\title{
Inferred calcification rate of a Mediterranean azooxanthellate coral is uncoupled with sea surface temperature along an $8^{\circ}$ latitudinal gradient
}

Erik Caroselli ${ }^{1}$, Guido Mattioli ${ }^{2}$, Oren Levy ${ }^{3}$, Giuseppe Falini ${ }^{4}$, Zvy Dubinsky ${ }^{3}$ and Stefano Goffredo ${ }^{1 *}$

\begin{abstract}
Introduction: Correlations between sea surface temperature (SST) and growth parameters of the solitary azooxanthellate Dendrophylliid Leptopsammia pruvoti were assessed along an $8^{\circ}$ latitudinal gradient on western Italian coasts (Mediterranean Sea), to check for possible negative effects of increasing temperature as the ones reported for a closely related, sympatric but zooxanthellate species.

Results: Calcification rate was correlated with skeletal density but not with linear extension rate, indicating that calcium carbonate deposition was preferentially allocated to keep a constant skeletal density. Unlike most studies on both temperate and tropical zooxanthellate corals, where calcification rate is strongly related to environmental parameters such as SST, in the present study calcification rate was not correlated with SST.

Conclusions: The lower sensitivity of L. pruvoti to SST with respect to other sympatric zooxanthellate corals, such as Balanophyllia europaea, may rely on the absence of a temperature induced inhibition of photosynthesis, and thus the absence of an inhibition of the calcification process. This study is the first field investigation of the relationship between SST and the three growth parameters of an azooxanthellate coral. Increasing research effort on determining the effects of temperature on biological traits of the poorly studied azooxanthellate scleractinians may help to predict the possible species assemblage shifts that are likely to occur in the immediate future as a consequence of global climatic change.
\end{abstract}

Keywords: Asymbiotic coral, Coral growth, Dendrophylliidae, Global warming, Scleractinia, Temperate coral

\section{Introduction}

Latitude is the main factor influencing the variation of light and temperature [1], two environmental parameters strongly linked to coral growth, physiology, demography and distribution pattern [2,3]. As a general trend, coral growth decreases with increasing latitude until a limit is reached where coral reef development no longer occurs, beyond $30^{\circ} \mathrm{N}$ and $30^{\circ} \mathrm{S}$ [4]. Coral growth can be defined by three related parameters (calcification = linear extension $\mathrm{x}$ skeletal density; [3,5]) whose measurement is essential when assessing the environmental effects on coral growth,

\footnotetext{
* Correspondence: s.goffredo@unibo.it

'Marine Science Group, Department of Biological, Geological and Environmental Sciences, Alma Mater Studiorum, University of Bologna, Via F. Selmi 3, Bologna, EU 40126, Italy

Full list of author information is available at the end of the article
}

because none of the three can perfectly predict the other two [6]. Analyzing these variables also allows predicting the possible effect of climatic changes on coral ecosystems $[7,8]$. These three variables have been studied in the field in the tropical genera Montastraea [5], Diploastrea [9], and Porites $[3,7,8,10]$, and their variation has been linked to changes in sea surface temperature (SST) and light associated with time and latitude. In colonies of $M$. annularis of the Gulf of Mexico and the Caribbean Sea, SST is positively correlated with calcification rate and skeletal density, while it is negatively correlated with linear extension rate [5]. In colonies of Porites of the Hawaiian archipelago, Thailand, and the Great Barrier Reef (Australia) SST is positively correlated with calcification and linear extension rates, and negatively correlated with skeletal density [5]. In

\section{Biomed Central}


contrast, monitoring efforts of 16 years of calcification in Porites colonies from the Great Barrier Reef [7] and 21 years of calcification in Porites colonies from Thailand [8] show that calcification declined over time, and suggests that the response may be due to the interactive effects of elevated seawater temperatures and $p \mathrm{CO}_{2}$ increase, as previously reported for colonies of Stylophora pistillata grown in aquaria [11]. However, a recent analysis of calcification of Porites colonies along an $11^{\circ}$ latitudinal gradient along Western Australia coasts has found no widespread patterns of decreasing calcification since 1900, and concludes that the main driver of change in coral calcification is the rate of change in the thermal environment of coral reefs [10].

In contrast with the large number of studies about the relationships between environmental parameters and coral growth in the tropics $[3,5,7,8,10]$, such studies are scarce for temperate zones. In Astrangia danae and Plesiastrea versipora, calcification rate increases with temperature, similarly to some tropical corals, albeit over a lower temperature range [12]. Laboratory observations on calcification rates in Cladocora caespitosa and Oculina patagonica show that long periods of elevated temperatures, corresponding to or higher than the maximum summer temperature in the field, lead to a decrease of calcification [13].

This study investigated the relationships between SST and the three growth components (calcification, skeletal density, and linear extension) in the temperate/ subtropical coral Leptopsammia pruvoti Lacaze-Duthiers, 1897. Leptopsammia pruvoti is an ahermatypic, nonzooxanthellate and solitary scleractinian coral, widely distributed in the Mediterranean basin and along the European Atlantic coast from Portugal through Southern England and Ireland [14]. It is one of the most common organisms in semi-enclosed rocky habitats, under overhangs, in caverns and small crevices at 0-70 $\mathrm{m}$ depth [14]. SST and solar radiation along an $850-\mathrm{km}$ latitudinal gradient on Western Italian coasts do not significantly influence its population abundance or skeletal architecture features such as corallite length, width, height [15], or its population structure stability and demographic traits [16]. However, the density of calcium carbonate crystals composing its skeleton (micro-density; [17]) is positively correlated with SST [18]. It is a gonochoric internal brooder [19], with a genetic structure characterized by heterozygote deficits at all scales, from patch to populations, without correlations between genetic differentiation and geographic distance, and with most genetic differentiation occurring between patches of the same study site, rather than between sites [20]. Its bright yellow colour and abundance makes this species attractive to recreational divers, who represent an important income for coastal tourist resorts in the Mediterranean Sea [21].
This is the first study on the variation of the three growth components in an azooxanthellate coral, and it aims to assess the variations of calcification rate, linear extension rate, and skeletal density in populations arranged along a latitudinal SST gradient. The results are also considered in the light of the most recent climate change scenarios and compared to the findings on the zooxanthellate Mediterranean endemic Dendrophylliiid coral Balanophyllia europaea.

\section{Results}

Mean annual SST varied significantly among the sites (Kruskal-Wallis test, $p<0.001$; Table 1 ). Mean skeletal density, linear extension and calcification rates were significantly different among the populations of Leptopsammia pruvoti (Kruskal-Wallis test, $p<0.001$; Table 2). To facilitate comparisons with published studies, the dependent and independent variables for the linear regression analyses between growth parameters (Table 3) were chosen according to literature data [3,5,22]. Mean skeletal density and calcification rate of the corallites in the populations were not correlated with mean linear extension rate (Table 3). Mean calcification rate of the corallites in the populations was positively correlated with mean skeletal density (Table 3). Based on the bootstrapping coefficients, calcification rate explained $67 \%$ of the variance in skeletal density (Table 3 ).

Considering the whole dataset (all ages), both the linear and power function models showed that none of the mean growth parameters of the populations were correlated with SST (Tables 4, 5). The lack of trends from the whole dataset was confirmed by the agestratified analyses on the subsets of immature, mature, and old samples (Tables 4,5). Thus, the mean growth parameters significantly differed among study sites, but their variation was not related to SST.

\section{Discussion}

The 'stretching modulation of skeletal growth' is a mechanism allowing corals to preferentially invest calcification resources in thickening the skeleton, thus increasing skeletal density, or accelerating linear extension $[5,23]$.

Table 1 Average annual solar radiation and SST values of the sample sites

\begin{tabular}{lcc}
\hline Population & Code & SST $\left({ }^{\circ} \mathrm{C}\right)$ annual mean (SE) \\
\hline Calafuria & $\mathrm{CL}$ & $18.02(0.04)$ \\
Elba & $\mathrm{LB}$ & $18.74(0.04)$ \\
Palinuro & $\mathrm{PL}$ & $19.14(0.03)$ \\
Scilla & $\mathrm{SC}$ & $19.54(0.02)$ \\
Genova & $\mathrm{GN}$ & $19.56(0.04)$ \\
Pantelleria & $\mathrm{PN}$ & $19.88(0.04)$ \\
\hline
\end{tabular}

The sites are arranged in order of increasing SST. 
Table 2 Leptopsammia pruvoti. Mean skeletal density, linear extension, and calcification rates values of the populations

\begin{tabular}{|c|c|c|c|c|c|c|c|c|}
\hline Population & Code & $n$ & $\begin{array}{c}\text { Average skeletal } \\
\text { density }\left(\mathrm{mg} \mathrm{mm}^{-3}\right)\end{array}$ & SE & $\begin{array}{c}\text { Average linear extension } \\
\text { rate }\left(\mathrm{mm} \mathrm{yr}^{-1}\right)\end{array}$ & SE & $\begin{array}{c}\text { Average calcification } \\
\text { rate }\left(\mathrm{mg} \mathrm{mm}^{-2} \mathrm{yr}^{-1}\right)\end{array}$ & SE \\
\hline Calafuria & $\mathrm{CL}$ & 210 & 1.56 & 0.07 & 0.79 & 0.01 & 1.26 & 0.06 \\
\hline Elba & LB & 76 & 1.07 & 0.07 & 0.61 & 0.02 & 0.76 & 0.06 \\
\hline Palinuro & $P L$ & 152 & 1.38 & 0.08 & 0.74 & 0.01 & 1.14 & 0.06 \\
\hline Scilla & SC & 115 & 1.50 & 0.07 & 0.69 & 0.02 & 1.00 & 0.05 \\
\hline Genova & GN & 123 & 1.31 & 0.09 & 0.61 & 0.01 & 1.08 & 0.07 \\
\hline Pantelleria & PN & 144 & 1.14 & 0.03 & 0.68 & 0.01 & 0.71 & 0.01 \\
\hline
\end{tabular}

The sites are arranged in order of increasing SST. $n$ number of individuals, SE standard error.

The tropical Porites, for example, invests increased calcification at higher temperatures into linear extension $[3,8]$. In contrast, the tropical Montastraea annularis invests increased calcification at higher temperatures to construct denser skeletons [5,23]. In the Mediterranean endemic Balanophyllia europaea, calcification is allocated evenly between increasing skeletal density and linear extension, indicating that the ability to colonize the substratum quickly and the mechanical strength of the skeleton are both important for this species [22]. The temperate L. pruvoti exhibited a response which was similar to the one of $M$. annularis, in that calcification was positively correlated with skeletal density but not with linear extension. For each $1 \mathrm{mg}$ $\mathrm{mm}^{-2} \mathrm{yr}^{-1}$ of calcification rate variation, skeletal density varied by $\sim 1 \mathrm{mg} \mathrm{mm}^{-3}$.

Geometrically calculated skeletal density values in the present work were reasonable with respect to other studies on tropical and temperate species $[3,5,17,22]$. The computed skeletal density used in this and in previous studies [15,22] is analogous to the bulk density [17], which is defined as the skeletal mass divided by the total volume (skeletal matrix volume plus pores volume; [17]). Skeletal matrix volume is further composed by the crystals of $\mathrm{CaCO}_{3}$ and by the intracrystalline organic matrix regulating the crystallization process [24]. Analyses to quantify the porosity in the same samples of the present study show that the variation of bulk density depends on variations of porosity, while the variation in the density of the skeletal framework (micro-density, [17]) is not strong enough to significantly affect bulk density [18].

The lack of correlations with SST exhibited by the calcification rate and skeletal density in the present study on Leptopsammia pruvoti confirms previous studies on the population density, growth and population structure stability of this species, where the coral parameters were always shown to be unrelated to environmental variables such as solar radiation or SST $[15,16]$. For both the linear and power function models, trends of the analyses performed on the full dataset were confirmed by the analyses on the three age-based subsets, indicating that differences in the mean age of the samples in the populations [16] did not bias the results.

The lack of correlation between calcification rate of the azooxanthellate L. pruvoti and SST along the latitudinal gradient is a different response with respect to the similar studies on temperate and tropical zooxanthellate species. For example, calcification rate of the Mediterranean endemic $B$. europaea is negatively related to SST [22], while in the tropical Porites and M. annularis it is positively related to SST $[3,5]$. However, mid-term studies on Porites highlight a reduction of its calcification rate as SST increases $[7,8]$, even if a recent long-term analysis of Porites calcification along Australian coasts show no evidence of widespread patterns of decline in calcification rate since 1900 [10]. In that analysis, calcification rates at high-latitude reefs were found to be more sensitive to temperature increase than more tropical reefs [10]. Another recent analysis of Porites spp. and Montastraea spp. in the Great Barrier Reef and Mexican Caribbean highlighted a negative response of calcification to increasing SST for both genera, but a higher sensitivity to temperature increase for the former genus, rather than the latter one [25]. This has fundamental consequences in light of future global warming scenarios, since differential reduction of calcification between

Table 3 Leptopsammia pruvoti

\begin{tabular}{|c|c|c|c|c|c|c|c|}
\hline Dependent variable & Independent variable & Slope (SE) & Intercept (SE) & $r^{2}$ & $r$ & $r_{B S}^{2}$ & $r_{B S}$ \\
\hline Skeletal density & Linear extension & - & - & 0.518 & 0.720 & 0.457 & 0.676 \\
\hline Calcification & Linear extension & - & - & 0.396 & 0.629 & 0.347 & 0.589 \\
\hline Calcification & Skeletal density & 0.969 & -0.294 & 0.753 & $0.868^{*}$ & 0.666 & $0.816^{*}$ \\
\hline
\end{tabular}

Linear regression and correlation analysis between mean skeletal density, linear extension rate, and calcification rate in the six sites $(n=6)$. $r^{2}$ Pearson's coefficient of determination, $r$ Pearson's correlation coefficient, $r_{B S}^{2}$ and $r_{B S}$ Pearson's coefficients calculated with bootstrapping, ${ }^{*} p<0.05$. SE standard error. 
Table 4 Leptopsammia pruvoti

\begin{tabular}{lcccc}
\hline Dependent variable & $r^{2}$ & $\boldsymbol{r}$ & $\boldsymbol{r}_{\boldsymbol{B S}}^{2}$ & $\boldsymbol{r}_{\boldsymbol{B S}}$ \\
\hline All samples & & & & \\
Skeletal density & 0.126 & -0.355 & 0.073 & -0.271 \\
Linear extension & 0.250 & -0.500 & 0.143 & -0.378 \\
Calcification & 0.261 & -0.510 & 0.181 & -0.426 \\
Immature samples (0-4 years) & & & \\
Skeletal density & 0.018 & -0.133 & 0.0003 & -0.018 \\
Linear extension & 0.013 & -0.115 & 0.017 & -0.129 \\
Calcification & 0.178 & -0.422 & 0.129 & -0.359 \\
Mature samples (5-8 years) & & & & \\
Skeletal density & 0.049 & -0.221 & 0.030 & -0.174 \\
Linear extension & 0.077 & -0.278 & 0.014 & -0.118 \\
Calcification & 0.016 & -0.126 & 0.008 & -0.090 \\
Old samples (>8 years) & & & & \\
Skeletal density & 0.301 & 0.548 & 0.274 & 0.523 \\
Linear extension & 0.148 & -0.384 & 0.122 & -0.349 \\
Calcification & 0.181 & 0.425 & 0.091 & 0.302 \\
\hline
\end{tabular}

Linear model. Correlation analysis between SST and growth parameters in the six sites $(n=6)$. No correlation resulted significant. ${ }^{2}$ Pearson's coefficient of determination, $r$ Pearson's correlation coefficient, $r_{B S}^{2}$ and $r_{B S}$ Pearson's coefficients calculated with bootstrapping.

coral genera could profoundly affect community structure [25]. Our results suggest a higher sensitivity of zooxanthellate species to the variations of temperature, while asymbiotic corals may be more tolerant to

Table 5 Leptopsammia pruvoti

\begin{tabular}{lcccc}
\hline Dependent variable & $r^{2}$ & $r$ & $r_{B S}^{2}$ & $r_{B S}$ \\
\hline All samples & & & & \\
Skeletal density & 0.105 & -0.324 & 0.067 & -0.258 \\
Linear extension & 0.225 & -0.474 & 0.133 & -0.365 \\
Calcification & 0.223 & -0.472 & 0.165 & -0.406 \\
Immature samples (0-4 years) & & & \\
Skeletal density & 0.032 & -0.178 & 0.0004 & -0.022 \\
Linear extension & 0.013 & -0.114 & 0.012 & -0.111 \\
Calcification & 0.204 & -0.451 & 0.123 & -0.351 \\
Mature samples (5-8 years) & & & & \\
Skeletal density & 0.051 & -0.225 & 0.021 & -0.146 \\
Linear extension & 0.077 & -0.277 & 0.013 & -0.112 \\
Calcification & 0.014 & -0.119 & 0.004 & -0.065 \\
Old samples (>8 years) & & & & \\
Skeletal density & 0.299 & 0.546 & 0.299 & 0.546 \\
Linear extension & 0.143 & -0.378 & 0.113 & -0.336 \\
Calcification & 0.268 & 0.518 & 0.144 & -0.380 \\
\hline Pow functon model(eqn & 2 . Lin
\end{tabular}

Power function model (eqn 2). Linear regression and correlation analysis between SST and growth parameters in the six sites $(n=6)$ calculated on logtransformed data. No correlation resulted significant. $r^{2}$ Pearson's coefficient of determination, $r$ Pearson's correlation coefficient, $r_{B S}^{2}$ and $r_{B S}$ Pearson's coefficients calculated with bootstrapping. temperature variations. The higher sensitivity of symbiotic species may be due to the decrease of photosynthetic performance at higher temperatures, since in zooxanthellate corals calcification is enhanced by photosynthesis [26], and both processes have temperature optima [12]. Alternatively, a role may be played by the much steeper response of respiration to subtle temperature increases $\left(\mathrm{Q}_{10}\right)$ than that of photosynthesis, resulting in significant decrease of the residual net photosynthesis and of the energy surplus needed for calcification and other physiological processes [27]. Although the hypothesis of photosynthetic inhibition at high temperatures is intriguing, other environmental parameters may influence coral calcification ( $\mathrm{pH}$, total alkalinity, wave exposition, flow rate, etc.). Besides local factors, the apparent insensitivity of $L$. pruvoti growth to the SST range experienced in the present study may be due either to 1 ) the lack of zooxanthellate, and thus a lack of inhibition of calcification by the depressed net photosynthesis, or 2) a higher optimal temperature for the calcification of this species with respect to B. europaea, or 3) a coupling between the above two factors, or 4) a sampling area not representative of the species conditions at the collection sites. L. pruvoti distribution area includes also regions outside the Mediterranean Sea, up to the southern coasts of Ireland and UK, where seawater temperature is considerably lower [14]. It is then unlikely that this species has a higher optimal temperature for calcification than the Mediterranean endemic B. europaea, since L. pruvoti lives in much colder seas and deeper waters (up to $70 \mathrm{~m}$ depth). Even if any comparison between $L$. pruvoti and B. europaea must be taken cautiously, since the two species were sampled at different depths (16 m and $6 \mathrm{~m}$, respectively), which may be subject to different thermal regimes throughout the year, the variation of calcification rate among sites, found in L. pruvoti, could be related to particular local conditions unrelated to temperature. Since the present study focused on the influence of SST, we selected sites with similar environmental traits other than SST, but we did not thoroughly analyze all the site characteristics such as nutrients and zooplankton availability or competitive interactions with other organisms, which could all contribute to the observed differences in calcification rate. However, these local differences, while contributing to the variability of calcification rate (this study) and of population dynamics traits [16], are not strong enough to determine significant variations in population abundance, which is homogeneous across all sites with about 10,000 individuals per square meter [15]. It may be argued that no correlation with SST has been found because the selected sampling area for this study was too small and unrepresentative of the population. However, the same sampling area adequately represents the sites in previous studies on the biometry, growth and population dynamics 
of the species $[15,16,22]$, where trends in the biometric parameters (such as polyp length) with temperature have been found [15]. Moreover, significant differences in calcification rate among sites have actually been found in the present study, but they do not correlate to temperature, and are likely due to local differences in parameters other than temperature. An alternative explanation of the difference in demographic parameters among sites may be related to suspension feeding. In the Mediterranean, the warm summer-fall season is characterized by lower nutrient levels and zooplankton availability than the cool winter-spring season [28]. Corals and several benthic suspension feeding taxa have proved to be stressed by low nutrients and limited zooplankton availability [28]. Different availability of resources among sites may affect calcification rate in L. pruvoti. However, if this was the case, negative effects on calcification rate would be expected in the warmest sites (where the warm season is longer and the zooplankton availability lower, on average). Instead, $L$. pruvoti calcification seems to be unrelated to SST. The differences in calcification rates and population dynamics traits among sites may be related to other environmental parameters not considered in this study $(\mathrm{pH}$, total alkalinity, wave exposition, flow rate, etc.). Further investigations are thus needed to better constrain the environmental controls on the population dynamics of this species. Moreover, further investigation on the poorly studied azooxanthellate species are needed to differentiate the environmental controls on the growth of symbiotic and asymbiotic corals.

One of the main threats for coral and coral reefs survival is global temperature increase [29]. The speeds of many negative changes to the oceans are near or are tracking the worst-case scenarios from the IPCC and other predictions [30]. Recently, one of the most diverse communities in the Mediterranean Sea, the coralligenous ( 1,666 species; [31]), where suspension feeders are dominant, has been strongly affected by several mass mortality events related to high temperatures [32-36]. The zooxanthellate dendrophylliid $B$. europaea is a Mediterranean endemic species which will likely be negatively affected by seawater warming, since increasing temperature lowers its population abundance, its skeletal density [15], by increasing its skeletal porosity [18], and lowers its calcification rate [22]. Moreover, warmer populations are less stable and show a progressive deficiency of young individuals, so that there is concern for the future of this species [37]. These detrimental effects of increasing temperature seem to be related to the symbiosis with zooxanthellae, whose photosynthesis could be depressed at high temperatures causing cascading negative effects on the growth and reproductive traits of B. europaea, although this hypothesis is yet to be tested $[15,18,22,37]$. L. pruvoti, instead, seems to be tolerant to the same temperature range experienced by $B$. europaea.
In fact, biological traits of the former species have been studied in the same sites and time interval, but none of them is negatively correlated with SST $([15,16,18]$ and present study). Increasing temperature may even favour $L$. pruvoti, since the corals living in populations characterized by higher SSTs have a higher micro-density, even if this increase in micro-density is not strong enough to cause significant variations of bulk density [18]. However, the limit of temperature increase that will still be tolerable by this species is unknown. Moreover, it should be noted that the results derived from analyses based on latitudinal variations of calcification are not necessarily the same as those derived from time-based analyses. In fact, while calcification may have a positive correlation with SST along a latitudinal gradient, such as in Porites [3], it may be negatively correlated with the increasing SST recorded in recent years $[7,8]$, and may fluctuate during the yearly cycle of temperature variation [38]. Thus, any extrapolations of spatial derived data to time resolved predictions has to be taken cautiously.

\section{Conclusions}

Unlike the zooxanthellate B. europaea, the differences in growth and population dynamics traits of the azooxanthellate $L$. pruvoti seem unrelated to SST along a wide latitudinal gradient in the Mediterranean Sea. These findings confirm previous observations that two species belonging to the same family and sharing a wide part of their distribution area may have very different temperature tolerance and consequent response to seawater warming [16]. The higher tolerance of L. pruvoti, relative to B. europaea, may indeed rely on the absence of symbionts, and thus the lack of an inhibition of host physiological processes by the heat-stressed zooxanthellae.

This study is the first field investigation of the relationship between SST and the three growth parameters of an azooxanthellate coral. Increasing research effort on determining the effects of temperature on biological traits of the poorly studied azooxanthellate scleractinians may help to predict the possible species assemblage shifts that are likely to occur in the immediate future as a consequence of global climatic change.

\section{Materials and methods}

Specimens of Leptopsammia pruvoti were collected from six sites along a latitudinal gradient, from $44^{\circ} 20^{\prime} \mathrm{N}$ to $36^{\circ}$ $45^{\prime} \mathrm{N}$, between 9 November 2003 and 30 September 2005 (Figure 1). Sampling sites were selected along the gradient to be characterized by different SST, which is the environmental parameter considered in this study and that has already shown correlations with biologic parameters of L. pruvoti in previous studies [15,18]. Samples were collected in each site using transects of three triangular patches of base $\times$ height equal to 


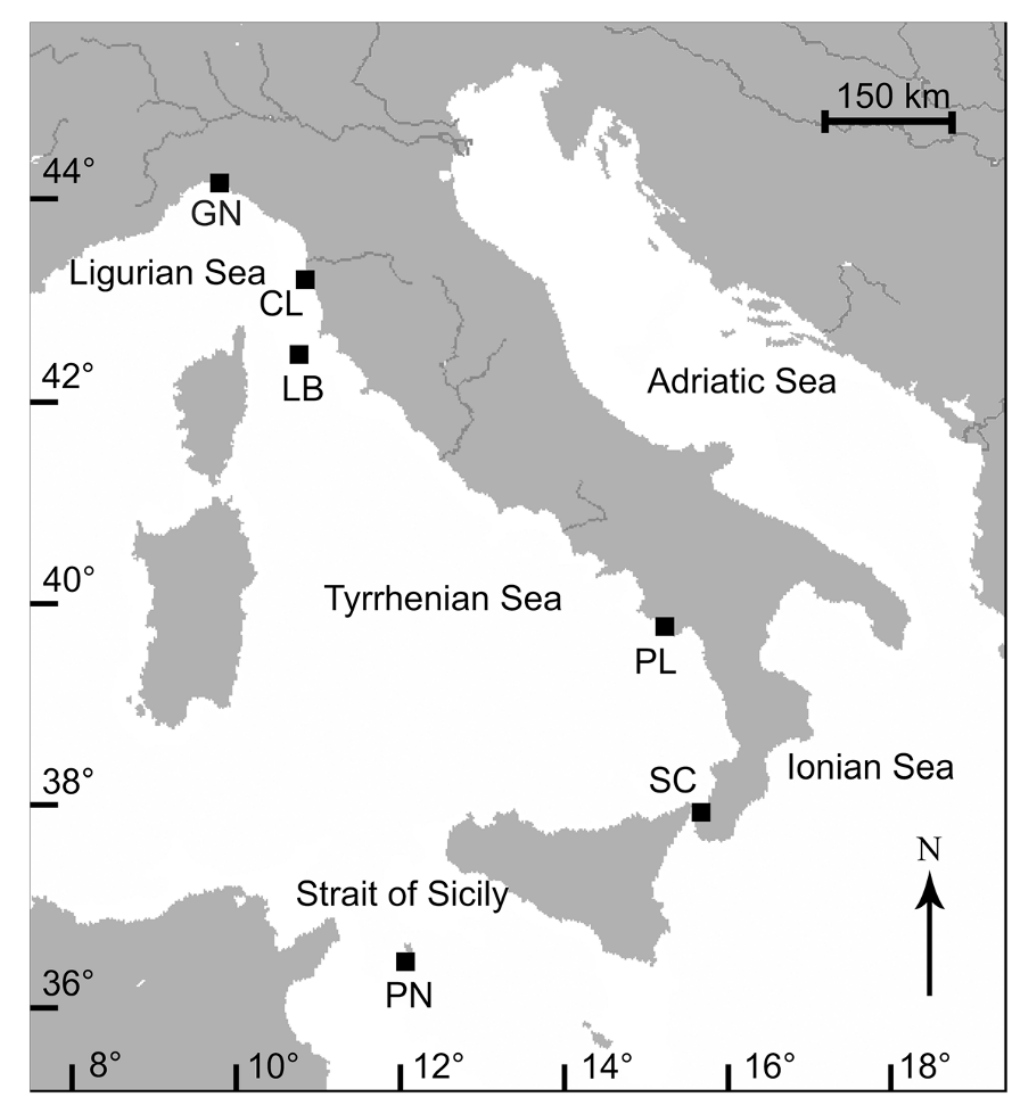

Figure 1 Map of the Italian coastline indicating sites where corals were collected. Abbreviations and coordinates of the sites in decreasing

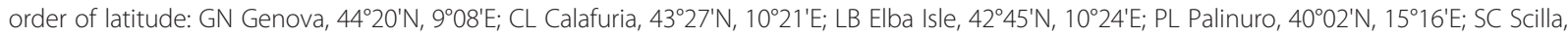
$38^{\circ} 01^{\prime} \mathrm{N}, 15^{\circ} 38^{\prime} \mathrm{E}$; PN Pantelleria Isle, $36^{\circ} 45^{\prime} \mathrm{N}, 11^{\circ} 57^{\prime} \mathrm{E}$.

$12 \mathrm{~cm} \times 7.1 \mathrm{~cm}$ (single patch area $=42.6 \mathrm{~cm}^{2}$; transect area per each site $=$ at least $42.6 \times 3=$ at least $128 \mathrm{~cm}^{2}$; [16]). Triangular patches were more easily placed in the narrow crevices colonized by the species, with respect to traditional square patches. Such a small patch area was chosen because of the high population density of the species (about 10,000 individuals $\mathrm{m}^{-1}$ ) which makes the sampling of all individuals present in larger areas (such as $1 \mathrm{~m}^{2}$ ) unfeasible [15]. Moreover, such sampling area is considered representative of the studied site in previous studies of the biometry, growth and population dynamics of this species, where significant differences among sites and correlations with SST have been found $[15,16,39]$. Sampling was performed at depths known to have high population densities and where the reproductive biology, biometry, population density, growth, population dynamics, and genetics of the species had previously been studied [15,16,19,20,39]. Patches were collected on the vault of crevices $3 \mathrm{~m}$ apart, at a depth of 15-17 m. All crevices were clearly separated one from each other, without a continuous presence of polyps from one patch to each other. In order to account for the high within-site genetic variation characterizing the species [20], it was necessary to sample different patches at each site and treat them as replicates, to have a meaningful picture of the growth parameters at each site. Because of the random distribution pattern of the species, the problems associated with regularly spaced quadrats and transects do not apply to this study [15]. All of the polyps present in each patch were collected.

Corals were dried at $50^{\circ} \mathrm{C}$ for four days and observed under a binocular microscope to remove fragments of substratum and calcareous deposits produced by other organisms. Corallite length $(L$ : maximum axis of the oral disc), width ( $W$ : minimum axis of the oral disc) and height ( $h$ : oral-aboral axis) were measured with calipers and the dry skeletal mass $(M)$ was measured with a precision balance. Corallite volume $(V)$ was determined by applying the formula: $V=L^{2} \mathrm{x} W^{2} \mathrm{x} h \pi$ [15]. Skeletal density (D) was calculated by dividing $M$ by $V$.

The age of each sample was estimated using the von Bertalanffy length-age growth function previously obtained and based on growth bands analysis by means of computerized tomography $[37,40]$. According to the age of the polyp, the annual linear extension rate was obtained for each sample using the von Bertalanffy 
length-age growth function $[16,40]$. The mean annual calcification rate (mass of $\mathrm{CaCO}_{3}$ deposited per year per area unit) was calculated for each sample by the formula: calcification $\left(\mathrm{mg} \mathrm{mm} \mathrm{mr}^{-1}\right)=$ skeletal density $\left(\mathrm{mg} \mathrm{mm}^{-3}\right) x$ linear extension $\left(\mathrm{mm} \mathrm{yr}^{-1}\right)[3,5,22]$. Thus, for each population the mean values of skeletal density, linear extension and calcification rates of the corallites were obtained. Samples were divided into three age classes: immature (0-4 years, after [16]); mature ( $4-8$ years, double the age at sexual maturity); old ( $>8$ years).

Correlation and regression analyses between environmental and growth parameters were performed both for the full dataset and for the three age classes, to check for differences due to the different mean age of the samples in the populations [12]. Relationships between environmental and growth parameters were performed using two models: a linear model and a power function model. The linear model was used to compare the results with other studies on environmental controls of coral growth, where linear functions are used [3,5]. Also the power function model was used as it produced the best fit with the data, and to compare the results obtained by the linear model. The power function model:

$$
y=\mathrm{a} x^{\mathrm{b}}
$$

was linearized with a log-transformation of both the independent and dependent variables, producing the equation:

$$
\ln (y)=\operatorname{bln}(x)+\ln (\mathrm{a})
$$

SST data for 2003-2005 were obtained for each location from the National Mareographic Network of the Agency for the Protection of the Environment and Technical Services (APAT, now renamed to Superior Institute for Environmental Research Protection, ISPRA, [41]). The data are measured by mareographic stations SM3810, built by the Italian Society for Precision Apparatuses (SIAP). Mean annual SST was obtained from hourly values measured from January 2001 to January 2005 (Table 1).

Because of the heteroskedastic nature of the data, the non-parametric Kruskal-Wallis test was used to compare mean SST, skeletal density, linear extension and calcification rates among the populations. Pearson correlation coefficients were calculated for the relationships among growth parameters and between environmental and growth parameters. Because of the low $n$ value $(n=6)$ and the assumptions of the Pearson method, correlation coefficients were also estimated with bootstrapping [42], with 100,000 resamples. All analyses were computed using PASW 18.0.

\section{Competing interests}

The authors declare that they have no competing interests.

\section{Authors' contributions}

SG conceived and designed the experiments. EC and GM performed the experiments. EC analyzed the data. EC, GM, OL, GF, ZD and SG wrote the paper. All authors read and approved the final manuscript.

\section{Acknowledgements}

The research leading to these results has received funding from the European Research Council under the European Union's Seventh Framework Programme (FP7/2007-2013) / ERC grant agreement n² 49930 - CoralWarm: Corals and global warming: the Mediterranean versus the Red Sea. This research was also financed by the Associazione dei Tour Operator Italiani (ASTOI), the Marine \& Freshwater Science Group Association, and the Ministry of Education, University and Research (MIUR). We wish to thank L. Bortolazzi, A. Comini, M. Ghelia, L. Tomesani, Centro Immersioni Pantelleria, II Pesciolino, Polo Sub, and Submaldive, Bologna Scuba Team, Scientific Diving School and Marine Science Group for help and assistance. Two anonymous reviewers gave comments which improved manuscript quality. The experiments complied with current Italian law.

\section{Author details}

${ }^{1}$ Marine Science Group, Department of Biological, Geological and Environmental Sciences, Alma Mater Studiorum, University of Bologna, Via F. Selmi 3, Bologna, EU 40126, Italy. ${ }^{2}$ Operative Unit of Radiology and Diagnostics by Images, Hospital of Porretta Terme, Local Health Enterprise of Bologna, Via Roma 16 Porretta Terme, Bologna, EU 40046, Italy. ${ }^{3}$ The Mina and Everard Goodman Faculty of Life Sciences, Bar-Ilan University, Ramat-Gan 52900, Israel. “Department of Chemistry "G. Ciamician", Alma Mater Studiorum, University of Bologna, Via F. Selmi 2, Bologna, EU 40126, Italy.

Received: 4 October 2012 Accepted: 6 November 2012

Published: 19 November 2012

\section{References}

1. Kain JM: The seasons in the subtidal. Br Phycol J 1989, 24:203-215.

2. Kleypas JA, McManus JW, Menez LAB: Environmental limits to coral reef development: where do we draw the line? Am Zool 1999, 39:146-159.

3. Lough JM, Barnes DJ: Environmental controls on growth of the massive coral Porites. J Exp Mar Biol Ecol 2000, 245:225-243.

4. Kinsey DW, Davies PJ: Carbon turnover, calcification and growth in coral reefs. In Biogeochemical cycling of mineral forming elements. Edited by Trudinger PA, Swaine DJ. Amsterdam: Elsevier; 1979:131-162.

5. Carricart-Ganivet JP: Sea surface temperature and the growth of the West Atlantic reef-building coral Montastraea annularis. J Exp Mar Biol Ecol 2004, 302:249-260.

6. Dodge RE, Brass GW: Skeletal extension, density and calcification of the reef coral, Montastrea annularis: St. Croix, U.S. Virgin Islands. Bull Mar Sci 1984, 34:288-307.

7. Cooper TF, De'ath G, Fabricius KE, Lough JM: Declining coral calcification in massive Porites in two nearshore regions of the northern Great Barrier Reef. Glob Change Biol 2008, 14:529-538.

8. Tanzil JTI, Brown BE, Tudhope AW, Dunne RP: Decline in skeletal growth of the coral Porites lutea from the Andaman Sea, South Thailand between 1984 and 2005. Coral Reefs 2009, 28:519-528.

9. Cantin NE, Cohen AL, Karnauskas KB, Tarrant AM, McCorkle DC: Ocean warming slows coral growth in the Central Red Sea. Science 2010, 329:322-325.

10. Cooper TF, O'Leary RA, Lough JM: Growth of Western Australian corals in the Anthropocene. Science 2012, 335:593-596.

11. Reynaud S, Leclercq N, Romaine-Lioud S, Ferrier-Pages C, Jaubert J, Gattuso JP: Interactive effects of $\mathrm{CO}_{2}$ partial pressure and temperature on photosynthesis and calcification in a scleractinian coral. Glob Change Biol 2003, 9:1660-1668

12. Howe SA, Marshall AT: Temperature effects on calcification rate and skeletal deposition in the temperate coral, Plesiastrea versipora (Lamarck). J Exp Mar Biol Ecol 2002, 275:63-81.

13. Rodolfo-Metalpa R, Richard C, Allemand D, Ferrier-Pagès C: Growth and photosynthesis of two Mediterranean corals, Cladocora caespitosa and 
Oculina patagonica, under normal and elevated temperatures. J Exp Biol 2006, 209:4546-4556.

14. Zibrowius $\mathrm{H}$ : Les scléractiniaires de la Méditeranée et de l'Atlantique nord-oriental. Mèm Inst Ocèanogr (Monaco) 1980, 11:1-284

15. Goffredo S, Caroselli E, Pignotti E, Mattioli G, Zaccanti F: Variation in biometry and population density of solitary corals with solar radiation and sea surface temperature in the Mediterranean Sea. Mar Biol 2007, 152:351-361.

16. Caroselli E, Zaccanti F, Mattioli G, Falini G, Levy O, Dubinsky Z, Goffredo S: Growth and demography of the solitary scleractinian coral Leptopsammia pruvoti along a sea surface temperature gradient in the Mediterranean Sea. PLOS ONE 2012, 7:e37848.

17. Bucher DJ, Harriott VJ, Roberts LG: Skeletal micro-density, porosity and bulk density of acroporid corals. J Exp Mar Biol Ecol 1998, 228:117-136.

18. Caroselli E, Prada F, Pasquini L, Nonnis Marzano F, Zaccanti F, Falini G, Levy O, Dubinsky Z, Goffredo S: Environmental implications of skeletal micro-density and porosity variation in two scleractinian corals. Zoology 2011, 114:255-264.

19. Goffredo S, Airi V, Radetić J, Zaccanti F: Sexual reproduction of the solitary sunset cup coral Leptopsammia pruvoti (Scleractinia, Dendrophylliidae) in the Mediterranean. 2. Quantitative aspects of the annual reproductive cycle. Mar Biol 2006, 148:923-932.

20. Goffredo S, Di Ceglie S, Zaccanti F: Genetic differentiation of the temperate-subtropical stony coral Leptopsammia pruvoti in the Mediterranean Sea. Isr J Ecol Evol 2009, 55:99-115.

21. Mundet $L$, Ribera L: Characteristics of divers at a Spanish resort. Tourism Manage 2001, 22:201-510.

22. Goffredo S, Caroselli E, Mattioli G, Pignotti E, Dubinsky Z, Zaccanti F: Inferred level of calcification decreases along an increasing temperature gradient in a Mediterranean endemic coral. Limnol Oceanogr 2009, 54:930-937.

23. Carricart-Ganivet JP, Merino M: Growth responses of the reef-building coral Montastraea annularis along a gradient of continental influence in the southern Gulf of Mexico. Bull Mar Sci 2001, 68:133-146.

24. Cohen AL, McConnaughey TA: Geochemical perspectives on coral mineralization. Rev Mineral Geochem 2003, 54:151-187.

25. Carricart-Ganivet JP, Cabanillas-Teran N, Cruz-Ortega I, Blanchon P: Sensitivity of calcification to thermal stress varies among genera of massive reef-building corals. PLOS ONE 2012, 7:e32859.

26. Al-Horani FA, Ferdelman T, Al-Moghrabi SM, de Beer D: Spatial distribution of calcification and photosynthesis in the scleractinian coral Galaxea fascicularis. Coral Reefs 2005, 24:173-180.

27. Al-Horani FA: Effects of changing seawater temperature on photosynthesis and calcification in the scleractinian coral Galaxea fascicularis, measured with $\mathrm{O}_{2}, \mathrm{Ca}^{2+}$ and $\mathrm{pH}$ microsensors. Sci Mar 2005, 69:347-354

28. Coma RM, Ribes M, Gili JM, Zabala M: Seasonality in coastal ecosystems. Trends Ecol Evol 2000, 12:448-453.

29. Hughes TP, Baird AH, Bellwood DR, Card M, Connolly SR, Folke C, Grosberg R, Hoegh-Guldberg O, Jackson JBC, Kleypas J, Lough JM, Marshall P, Nyström M, Palumbi SR, Pandolfi JM, Rosen B, Roughgarden J: Climate change, human impacts, and the resilience of coral reefs. Nature 2003, 301:929-933.

30. Rogers AD, Laffoley D'A: International Earth system expert workshop on ocean stresses and impacts. Summary report. Oxford: IPSO; 2011.

31. Ballesteros E: Mediterranean coralligenous assemblages: A synthesis of the present knowledge. Oceanogr Mar Biol Annu Rev 2006, 44:123-195.

32. Cerrano C, Bavestrello G, Bianchi CN, Cattaneo-Vietti R, Bava S, Morganti C, Morri C, Picco P, Sara G, Schiaparelli S, Siccardi A, Sponga F: A catastrophic mass-mortality episode of gorgonians and other organisms in the Ligurian Sea (NW Mediterranean), summer 1999. Ecol Lett 2000, 3:284-293.

33. Perez T, Garrabou J, Sartoretto S, Harmelin JG, Francour P, Vacelet J: Mass mortality of marine invertebrates: an unprecedented event in the Northwestern Mediterranean. CR Acad Sci III 2000, 323:853-865.

34. Rodolfo-Metalpa R, Bianchi CN, Peirano A, Morri C: Coral mortality in NW Mediterranean. Coral Reefs 2000, 19:24.

35. Coma R, Ribes M, Serrano E, Jimenez E, Salat J, Pascual J: Global warmingenhanced stratification and mass mortality events in the Mediterranean. Proc Natl Acad Sci U S A 2009, 106:6176-6181.

36. Garrabou J, Coma R, Bensoussan N, Bally M, Chevaldonne P, Cigliano M, Diaz D, Harmelin JG, Gambi MC, Kersting DK, Ledoux JB, Lejeusne C, Linares C,
Marschal C, Perez T, Ribes M, Romano JC, Serrano E, Teixido N, Torrents O, Zabala M, Zuberer F, Cerrano C: Mass mortality in the NW Mediterranean rocky benthic communities: Effects of the 2003 heat wave. Glob Change Biol 2009, 15:1090-1103.

37. Goffredo S, Caroselli E, Mattioli G, Pignotti E, Zaccanti F: Relationships between growth, population structure and sea surface temperature in the temperate solitary coral Balanophyllia europaea (Scleractinia, Dendrophylliidae). Coral Reefs 2008, 27:623-632.

38. Carricart-Ganivet JP: Coral skeletal extension rate: an environmental signal or a subject to inaccuracies? J Exp Mar Biol Ecol 2011, 405:73-79.

39. Goffredo S, Caroselli E, Mattioli G, Zaccanti F: Growth and population dynamic model for the non-zooxanthellate temperate solitary coral Leptopsammia pruvoti (Scleractinia, Dendrophylliidae). Mar Biol 2010 157:2603-2612.

40. von Bertalanffy L: A quantitative theory of organic growth (inquiries on growth laws II). Hum Biol 1938, 10:181-213.

41. Superior Institute for Environmental Research Protection. http://www. isprambiente.gov.it

42. Efron B: Nonparametric estimates of standard error: the jackknife, the bootstrap and other methods. Biometrika 1981, 68:589-599.

doi:10.1186/1742-9994-9-32

Cite this article as: Caroselli et al:: Inferred calcification rate of a Mediterranean azooxanthellate coral is uncoupled with sea surface temperature along an $8^{\circ}$ latitudinal gradient. Frontiers in Zoology 2012 $9: 32$

\section{Submit your next manuscript to BioMed Central and take full advantage of:}

- Convenient online submission

- Thorough peer review

- No space constraints or color figure charges

- Immediate publication on acceptance

- Inclusion in PubMed, CAS, Scopus and Google Scholar

- Research which is freely available for redistribution 